\title{
Erythropoietin Inhibits Hypoxia-Induced Epithelial-To-Mesenchymal Transition via Upregulation of miR-200b in HK-2 Cells
}

\author{
Jiuxu Bai ${ }^{a}$ Xiao Xiao ${ }^{b}$ Xiaoling Zhang ${ }^{a}$ Hanmin Cui $^{a}$ Junfeng $\mathrm{Hao}^{a}$ \\ Jingming $\mathrm{Han}^{\mathrm{a}}$ Ning $\mathrm{CaO}^{\mathrm{a}}$ \\ aDepartment of Blood Purification, General Hospital of Shenyang Military Area Command, Shenyang, \\ bState Key Laboratory of Cancer Biology and Xijing Hospital of Digestive Diseases, Xijing Hospital, \\ Fourth Military Medical University, Xi'an, China
}

\section{Key Words}

Erythropoietin • miR-200b • Endothelial-to-mesenchymal transition • Renal tubular

\begin{abstract}
Background/Aims: Renal tubular epithelial-mesenchymal transition (EMT) is regarded as an important factor leading to renal interstitial fibrosis. Erythropoietin (EPO) has been reported to attenuate renal fibrosis. The mechanism underlying this protective effect of EPO remains unclear. In this study, we aim to identify possible mechanisms of the EPO renoprotective effect. Methods: Hypoxia was induced in vitro by incubating human proximal tubular epithelial cell line $\mathrm{HK}-2$ cells in $1 \% \mathrm{O}_{2}$ and $5 \% \mathrm{CO}_{2}$. Western blotting and reverse transcription polymerase chain reaction analyses were used to evaluate the expression of epithelial and mesenchymal markers in the cell samples. The expression of miR-200b in the HK-2 cells under hypoxia or treatment with EPO was examined. Results: EPO represses hypoxia-induced EMT by upregulating miR-200b in HK-2 cells. Overexpression of miR-200b represses the effect of ETS proto-oncogene 1 (Ets-1)-induced EMT in HK-2 cells. Conclusion: miR-200 mediates the protective effects of EPO on EMT in hypoxic HK-2 cells. EPO attenuated hypoxia-induced EMT by increasing miR-200 expression via the repression of Ets-1.

\section{Introduction}

Renal interstitial fibrosis is a common outcome of end stage renal disease (ESRD). Chronic hypoxia is an important pathway that leads to end-stage renal failure, and it is also important in renal fibrogenesis [1]. The development of renal interstitial fibrosis in chronic kidney disease (CKD) is aggravated by chronic tubulointerstitial hypoxia. A previous study has suggested that chronic tubulointerstitial hypoxia induced by peritubular capillary loss aggravates the development of tubulointerstitial fibrosis in remnant kidneys

J. Bai and X. Xiao contributed equally to this work. 
[2]. Renal tubular epithelial-mesenchymal transition (EMT) is regarded as an important factor leading to renal interstitial fibrosis. Recent studies have suggested that tubular cells exposed to hypoxia undergo EMT, through transdifferentiation into myofibroblasts, which is characterized by the expression of mesenchymal markers and the acquisition of mobility [3, 4]. Other studies have indicated that hypoxia plays a important role in EMT which induces the development of renal fibrosis [5].

Erythropoietin (EPO) is a hematopoietic hormone produced primarily by the kidneys which promotes the formation of red blood cells by stimulating bone marrow. In addition to its hematopoietic effects, EPO has been shown to play an important role as a novel cytoprotective agent, which mediates anti-apoptosis and anti-inflammatory responses [6, 7]. EPO may be an efficient renoprotective agent against renal dysfunction, and has been shown to enhance functional recovery in rats with acute renal failure induced by ischemia and reperfusion [8]. Clinical studies have reported that EPO could retard kidney disease progression to chronic renal failure [9]. EPO has also been reported to play a role in attenuating the progression of renal tubulointerstitial fibrosis [10]. However, the precise mechanisms underlying the protective effects of EPO have not yet been fully elucidated.

miRNAs are 20-22 nt non-coding RNA molecules that regulate gene expression by interacting with the 3' untranslated region (UTR) of their respective mRNAs and induce translational suppression or degradation of mRNA [11]. miRNAs are involved in regulating diverse physiological processes, and certain miRNAs such as miR-29 and miR-200 have been shown to inhibit EMT and reduce fibrosis which is regulated by TGF- $\beta[12,13]$. Previous studies have suggested that the miR-200 family including miR-200a, miR-200b, miR-200c, miR-429 and miR-141 inhibit EMT through directly targeting zinc finger E-box-binding homeobox (ZEB)-1 and ZEB-2, which are E-cadherin transcriptional repressors [14]. To date, there are few studies that indicate the importance of miRNAs in the biological effects of EPO and the contribution of miRNAs to EPO-mediated mechanisms is still unknown [15].

In this study, it is shown that administration of EPO markedly attenuates EMT in human proximal tubular cells under hypoxic conditions. It is demonstrated that miR-200b is hypoxia-inducible which is regulated by EPO in hypoxic proximal tubular cells.

\section{Material and Methods}

\section{Cell culture}

The HK-2 human proximal tubular epithelial cell line was purchased from the American Type Culture Collection (Manassas, VA, USA) and cultured in Dulbecco's modified Eagle's medium/F12 medium (Invitrogen, Carlsbad, CA, USA) supplemented with 10\% fetal bovine serum (Gibco, Carlsbad, CA, USA). The cells were cultured at $37^{\circ} \mathrm{C}$ in a humidified atmosphere of $21 \% \mathrm{O}_{2}$ and $5 \% \mathrm{CO}_{2}$. Hypoxic conditions were created using Edwards Instruments in a hypoxic $\left(1 \% \mathrm{O}_{2}, 5 \% \mathrm{CO}_{2}, 37^{\circ} \mathrm{C}\right)$ incubator (Galaxy oxygen control incubator, RS Biotech, Irvine, UK). Experiments were undertaken after $48 \mathrm{~h}$ of hypoxic incubation, at which stage cell lysates and RNA were collected. The cells were kept in serum-free medium for $24 \mathrm{~h}$ and were treated with $10 \mathrm{U} / \mathrm{mL}$ EPO [16] recombinant human protein (Gibco, Frederick, BRL Co. Ltd., MD, USA) for an additional $48 \mathrm{~h}$.

Transfection of miR mimics, miR inhibitors, siRNA or Expressing Plasmid

hsa-miR-200b-5p (miR-200b) mimics (50 nM), hsa-miR-200b-5p inhibitor (100 nM), negative control (100 nM), Ets-1 siRNA (100 nM) [17] or pcDNA3.0-Ets-1 (GenePharma, Shanghai, China) were transfected into HK-2 cells using Lipofectamine 2000 (Invitrogen, Carlsbad, California) following the protocols provided by the manufacture. The cells were cultured for $24 \mathrm{~h}$ after transfection and then treated with $10 \mathrm{U} / \mathrm{mL}$ EPO and exposed to hypoxia for $48 \mathrm{~h}$.

RNA Isolation and Quantitative Real-time RT-PCR

Total RNA, including the mature miRNAs, of the cultured HK-2 cells was extracted using Trizol reagent (Invitrogen) according to the protocol supplied by the manufacturer. For mRNA, the reverse transcription 
reaction was performed using SuperScript ${ }^{\mathrm{TM}}$ II reverse transcriptase (Invitrogen). cDNA was detected using $\mathrm{SYBR}^{\circledR}$ Select Master Mix (Life technology, California, USA). For quantification of miRNAs, a miScript PCR Starter Kit (Qiagen, Hilden, Germany) was used, which consists of the miScript reverse transcription kit, miScript primer assays, and miScript SYBR Green PCR kit. GAPDH and U6 RNA were used as internal loading controls for mRNAs and miRNAs, respectively. After reactions, the Ct data were determined using default threshold settings, and the mean Ct was determined from the duplicate PCRs. The miR level of each sample was calculated by $2^{-\Delta \mathrm{Ct}}$, then $2^{-\Delta \Delta \mathrm{Ct}}$, which represented the fold change between two groups. $\Delta \mathrm{Ct}=\mathrm{Ct}{ }_{\text {miR value of }}$ target gene $-\mathrm{Ct}_{\text {value of internal referee U6 RNA }} \Delta \Delta \mathrm{Ct}=\Delta \mathrm{Ct}$ treatment $-\Delta \mathrm{Ct}$ control .

Luciferase reporter assay

For Ets-1 3'UTR luciferase reporter experiments, oligonucleotides harboring wild-type or mutated miR-200 binding sites from the human Ets-1 3'UTR were annealed and ligated into the EcoRI and PstI sites of the pGL3-control-mcs2 reporter vector (constructed by Wang Tao, PhD). HK-2 cells were seeded in a 48-well plate the day before transfection. HK-2 cells were cotransfected using the Ets-1 3'UTR reporter plasmid along with the pRL-TK Renilla plasmid (Promega, Madison, WI) and miR-200b mimics (50 nM) or a miRNA-negative control (50 nM). The miR-200b-200a-429 promoter firefly luciferase reporter assays were performed in a similar manner by using $100 \mathrm{ng}$ miR-200b-200a-429 promoter firefly luciferase reporter plasmid [18] with 200 ng pCMV5-Smad7 plasmids (Addgene, MA, USA) or Smad7 siRNA (GenePharma). Cells were harvested 48 hours later and measured using the Dual-Luciferase assay kit (Promega).

\section{Western blotting}

Western blotting was performed on cell lysates collected from HK-2 cells for detection of $\alpha$-SMA, E-cadherin and Ets-1. Proteins were separated on a Sodium dodecyl sulfate/Polyacrylamide gel electrophoresis gel (SDS/PAGE), transferred onto a polyvinylidene fluoride (PVDF) membrane and subjected to immunoblot analysis. Blotting was performed using antibodies against $\alpha$-SMA (1:500, Abcam, Cambridge, MA, USA), E-cadherin (1:500, Abcam), Ets-1 (1:500, Abcam) and GAPDH (1:10000, Abcam). After rinsing with Tris-buffered saline supplemented with $0.1 \%$ Tween 20 (TBST), the membranes were incubated with a horseradish peroxidase (HRP)-conjugated anti-rabbit or anti-goat antibody for $60 \mathrm{~min}$ at room temperature. The detection of specific signals was performed using enhanced chemiluminescence (ECL, Amersham Biosciences, Little Chalfont, UK).

\section{Immunofluorescence}

HK-2 cells, which were grown and stimulated in six-well chamber slides, were fixed using $3.7 \%$ formaldehyde for $10 \mathrm{~min}$ at $4^{\circ} \mathrm{C}$. The HK-2 cells membrane was permeabilized using $0.2 \%$ Triton X-100 for $5 \mathrm{~min}$. Then the cells were incubated with an anti- $\alpha$-SMA antibody overnight at $4^{\circ} \mathrm{C}$. After that, cells were exposed to a goat anti-rabbit Cy3 conjugated secondary antibody for $2 \mathrm{~h}$ at $37^{\circ} \mathrm{C}$.

\section{Statistical Analyses}

Data reported represent mean \pm SEM of at least three independent experiments. Statistical significance, defined as $P<0.05$, was evaluated using one-way analysis of variance with SPSS software (version 19.0).

\section{Results}

EPO Treatment Attenuated hypoxia-induced EMT in HK-2 Cells

Epithelial cells undergoing EMT acquire expression of mesenchymal markers such as $\alpha$-SMA and lose expression of epithelial markers such as E-cadherin. Hypoxia was induced in vitro by incubating $\mathrm{HK}-2$ cells in $1 \% \mathrm{O}_{2}$ and $5 \% \mathrm{CO}_{2}$. After $48 \mathrm{~h}$ of hypoxia, HK-2 cells underwent a morphological and phenoptypic transition characteristic of EMT, which was attenuated by EPO (Fig. 1A). The level of $\alpha$-SMA was significantly increased compared with levels in normoxic cells, whereas the E-cadherin protein was decreased in hypoxic cells $(\mathrm{P}<$ 0.05; Fig. 1B,C). Treatment with EPO attenuated the hypoxia-induced upregulation of $\alpha$-SMA. Expression of E-cadherin was correspondingly increased in hypoxic cells after treatment with EPO. Immunofluorescence detection of $\alpha$-SMA confirmed the results of the western blotting. These changes are all characteristic of myofibroblasts (Fig. 1D). 
Fig. 1. Erythropoietin (EPO) attenuates hypoxia-induced EMT in HK-2 cells. (A) Morphological changes in HK-2 cells were observed using an inverted microscope. (B) Real time PCR analyses for $\alpha$-SMA and E-cadherin in HK-2 cells after hypoxia and EPO treatment for $48 \mathrm{~h}$. The data were normalized using a GAPDH control. ${ }^{*} P<$ 0.05. (C) Western blot analysis of $\alpha$-SMA and E-cadherin in HK-2 cells after hypoxia and EPO (10 U/mL) treatment for $48 \mathrm{~h}$; $\beta$-Actin serves a loading control. (D) Immunofluorescence labeling of HK-2 cells after hypoxia and EPO (10 $\mathrm{U} / \mathrm{mL}$ ) treatment for 48 $h$ with an anti- $\alpha$-SMA antibody.

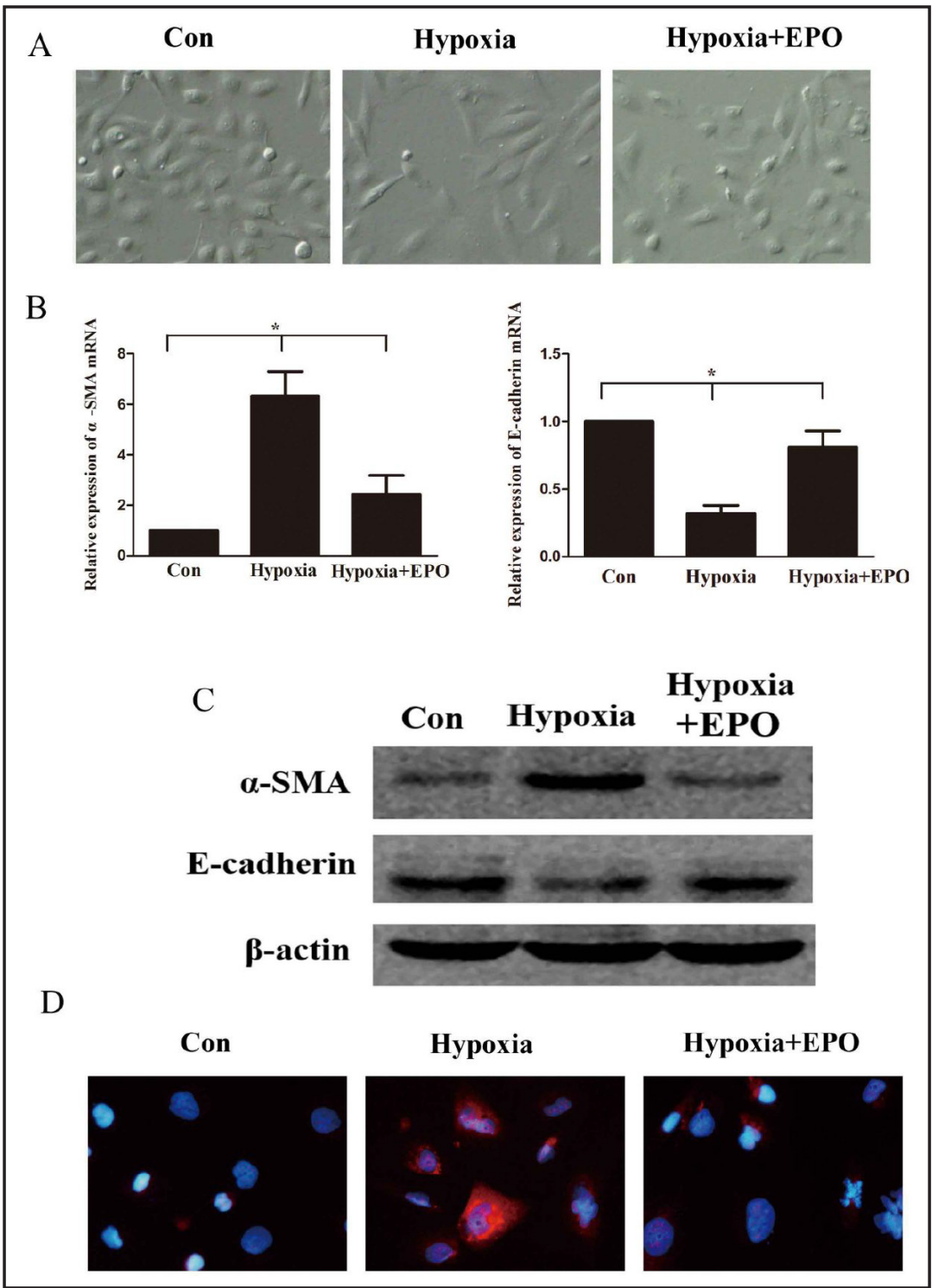

EPO represses hypoxia-induced EMT by upregulating miR-200b in HK-2 cells

To elucidate the underlying mechanism, the putative role of the miRNAs was investigated. miR-200 is a well-studied miRNA involved in EMT. Whether miR-200 was regulated in HK-2 cells under hypoxic conditions was investigated. Real-time PCR demonstrated that the expression of the miR-200 family including miR-200a, miR-200b and miR-429 decreased following $48 \mathrm{~h}$ of hypoxic conditions in HK-2 cells (Fig. 2A). miR-200 expression in EPOtreated HK-2 cells was then investigated. HK-2 cells were treated using $10 \mathrm{U} / \mathrm{mL}$ EPO for $48 \mathrm{~h}$. Analysis of miR-200 expression using real-time PCR showed that miR-200a, miR-200b and miR-429 expression increased in response to treatment with EPO in HK-2 cells (Fig. 2B). miR-200b is a miR-200 family member. Treatment with miR-200b significantly reduced the increase in the $\alpha$-SMA levels induced by hypoxia. The decreased levels of E-cadherin in HK-2 cells in hypoxic conditions were markedly attenuated by miR-200b treatment. To further verify that EPO attenuates EMT in hypoxic HK-2 cells by increasing miR-200b, a miR-200b inhibitor was used before treatment with EPO under hypoxic conditions. PCR and western blotting showed that the protective effect of EPO in attenuating EMT was eliminated by miR$200 \mathrm{~b}$ inhibition (Fig. 2C, D). Expression of $\alpha$-SMA was markedly increased and E-cadherin expression was markedly decreased by miR-200b inhibition in the EPO-treated hypoxic HK-2 cells. Immunofluorescence detection of $\alpha$-SMA confirmed the results of the western 
A

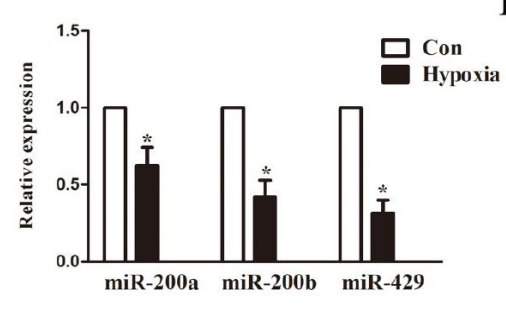

$\mathrm{C}$

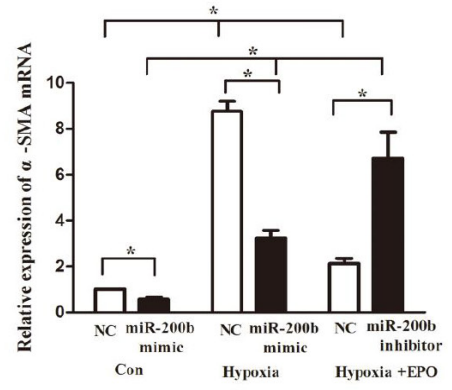

B
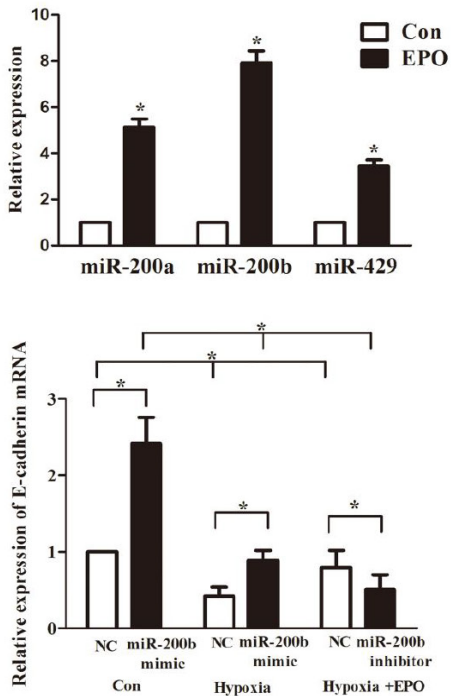

$\mathrm{D}$

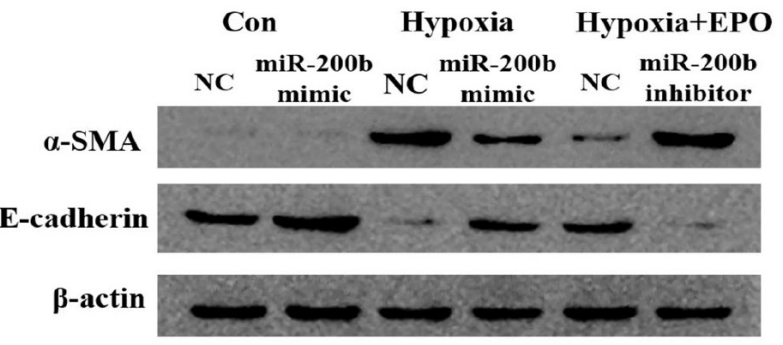

$\mathrm{E}$
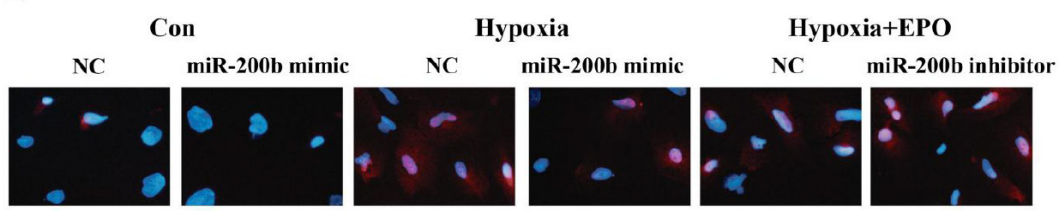

Fig. 2. EPO represses hypoxia-induced EMT by upregulating miR-200b in HK-2 cells. (A) Relative expressions of miR-200a, miR-200b, and miR-429 in HK-2 cells after hypoxia for $48 \mathrm{~h}$ were determined using real-time RT-PCR. miRNA expression was normalized to the RNU6B small nuclear ribonucleic acid (snRNA), and the results are expressed as fold change relative to control. ${ }^{*} P<0.05$ versus control (Con). (B) Relative expressions of miR-200a, miR-200b, and miR-429 in HK-2 cells induced by EPO (10 U/mL) for $48 \mathrm{~h}$ were determined using real-time RT-PCR. miRNA expressions were normalized to the RNU6B snRNA, and results are expressed as fold change relative to Con. ${ }^{*} P<0.05$ versus Con. (C) Real time PCR analyses for $\alpha$-SMA and E-cadherin expression in HK-2 cells treated with hypoxia $\left(1 \% \mathrm{O}_{2}\right)$ and EPO $(10 \mathrm{U} / \mathrm{mL})$ for $48 \mathrm{~h}$ with or without miR-200b mimics. The data were normalized using a GAPDH control. ${ }^{*} P<0.05$. (D) Western blot analysis of $\alpha$-SMA and E-cadherin in HK-2 cells treated with hypoxia $\left(1 \% \mathrm{O}_{2}\right)$ and EPO $(10 \mathrm{U} / \mathrm{mL})$ for $48 \mathrm{~h}$ with or without miR-200b mimics. $\beta$-Actin serves a loading control. (E) Immunofluorescence labeling of HK-2 cells treated with hypoxia $\left(1 \% \mathrm{O}_{2}\right)$ and EPO $(10 \mathrm{U} / \mathrm{mL})$ for $48 \mathrm{~h}$ with or without miR-200b mimics; cells were immunostained using an anti- $\alpha$-SMA antibody.

blotting (Fig. 2E). These results collectively suggest that upregulated miR-200b induced by EPO attenuated hypoxic HK-2 cells undergoing EMT.

EPO activated miR-200b-200a-429 transcription in a Smad7 dependent manner

To investigate the molecular mechanisms by which EPO upregulated miR-200b transcription, a series of miR-200b-200a-429 promoter were constructed, and the luciferase 


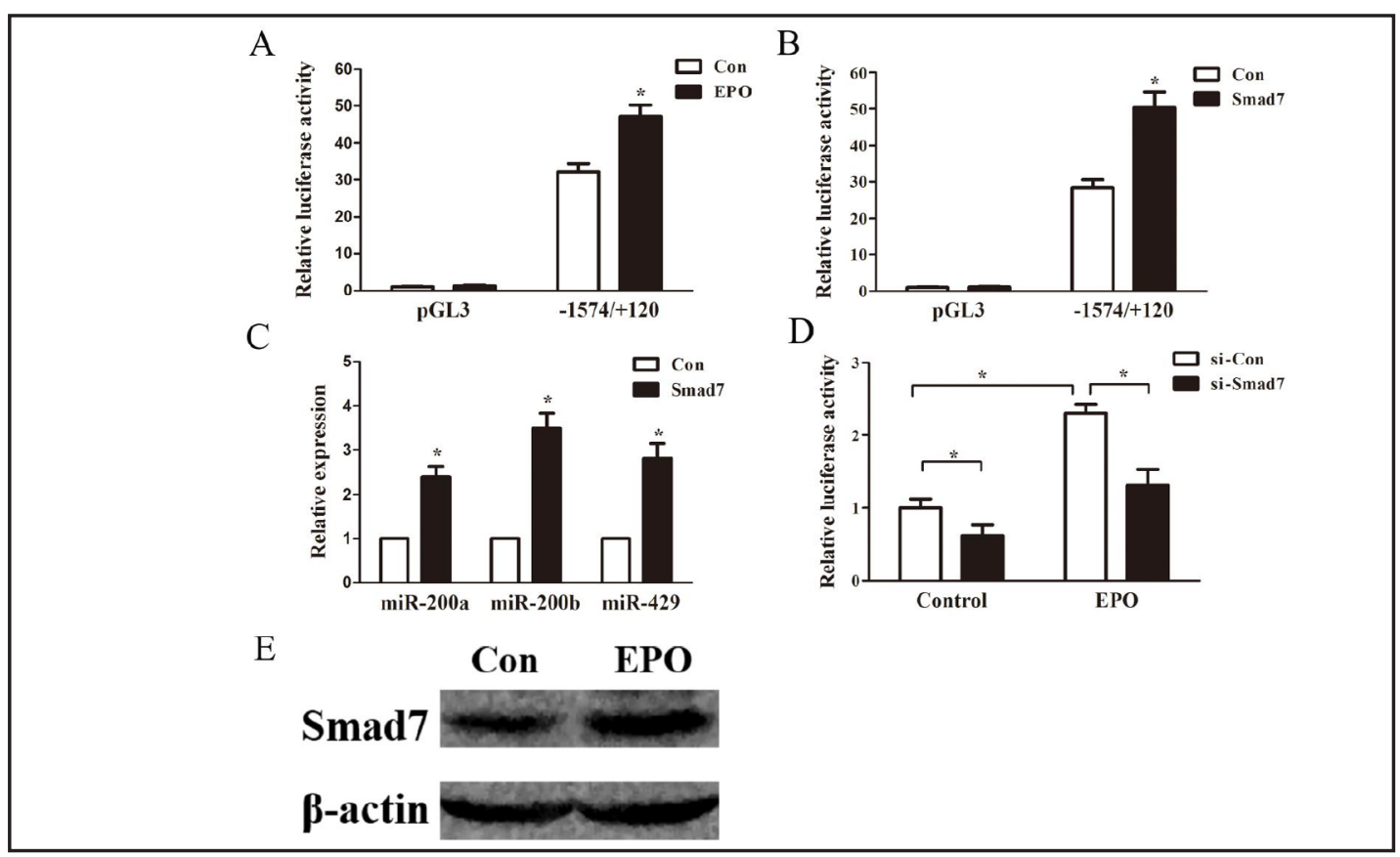

Fig. 3. EPO activated miR-200b-200a-429 transcription in a Smad7 dependent manner. (A) miR-200b200a-429 promoter activity was determined by luciferase reporter assay. HK-2 cells were seeded on 24-well plates and transfected with miR-200b-200a-429 promoter plasmids and treated with EPO for 48 hours. The firefly luciferase activity was normalized to that of the renilla luciferase. Values were expressed as mean \pm SEM. ${ }^{*} P<0.05$ versus control (Con). (B) HK-2 cells were cotransfected with promoter reporter constructs and Smad 7 expressing plasmid as indicated. Relative luciferase activity was determined 48 hours after transfection. ${ }^{*} P<0.05$ versus control (Con). (C) HK-2 cells were transfected with Smad7 expressing plasmids, and the expressions of miR-200a, miR-200b, and miR-429 was quantified by real-time PCR after 48 hours. ${ }^{*} P<0.05$ versus control (Con). (D) HK-2 cells were cotransfected with promoter reporter constructs and Smad7 siRNAs. Cells were treated with or without EPO for 48 hours after transfection. $* P<0.05$. (E) HK-2 cells were treated with EPO for 48 hours, Smad7 protein levels were assessed by Western blot.

activity of this construct was tested after transfection in HK-2 cells. EPO dramatically enhanced the luciferase activities of miR-200b-200a-429 promoter (Fig. 3A). As revealed previously, Smad7 completely blocked the TGF-1-mediated miR-200 family downregulation [19]. Therefore, we hypothesized that Smad7 may is involved in EPO activated miR-200b200a-429 transcription in HK-2 cells. To further demonstrate this, the promoter was cotransfected with Smad7 expressing plasmid into HK-2 cells. The results showed that Smad7 enhanced the activities of miR-200b-200a-429 promoter (Fig. 3B). Furthermore, overexpression of Smad7 also increased expressions of miR-200a, miR-200b, and miR-429 compared with control (Fig. 3C). We next investigated if EPO upregulated mir-200b-200a-429 transcription in a Smad7 dependent manner. In EPO-treated HK-2 cells, knockdown of Smad7 repressed the luciferase activity which was enhanced by EPO (Fig. 3D). To further confirm the contribution of Smad7 in EPO-induced mir-200b-200a-429 luciferas activity, we investigated the expression of Smad7 in EPO-treated HK-2 cells. The protein levels of Smad7 increased under EPO treatment compared with control (Fig. 3E). Taken together, these results indicate that Smad7 is an important mediator in EPO activated miR-200b-200a-429 transcription.

EPO represses hypoxia-induced EMT by decreasing Ets-1 in HK-2 cells

Ets-1 is an important transcription factor recognized as an important mediator of fibrosis [20]. Whether Ets-1 is involved in inducing EMT in HK-2 cells under hypoxic conditions 


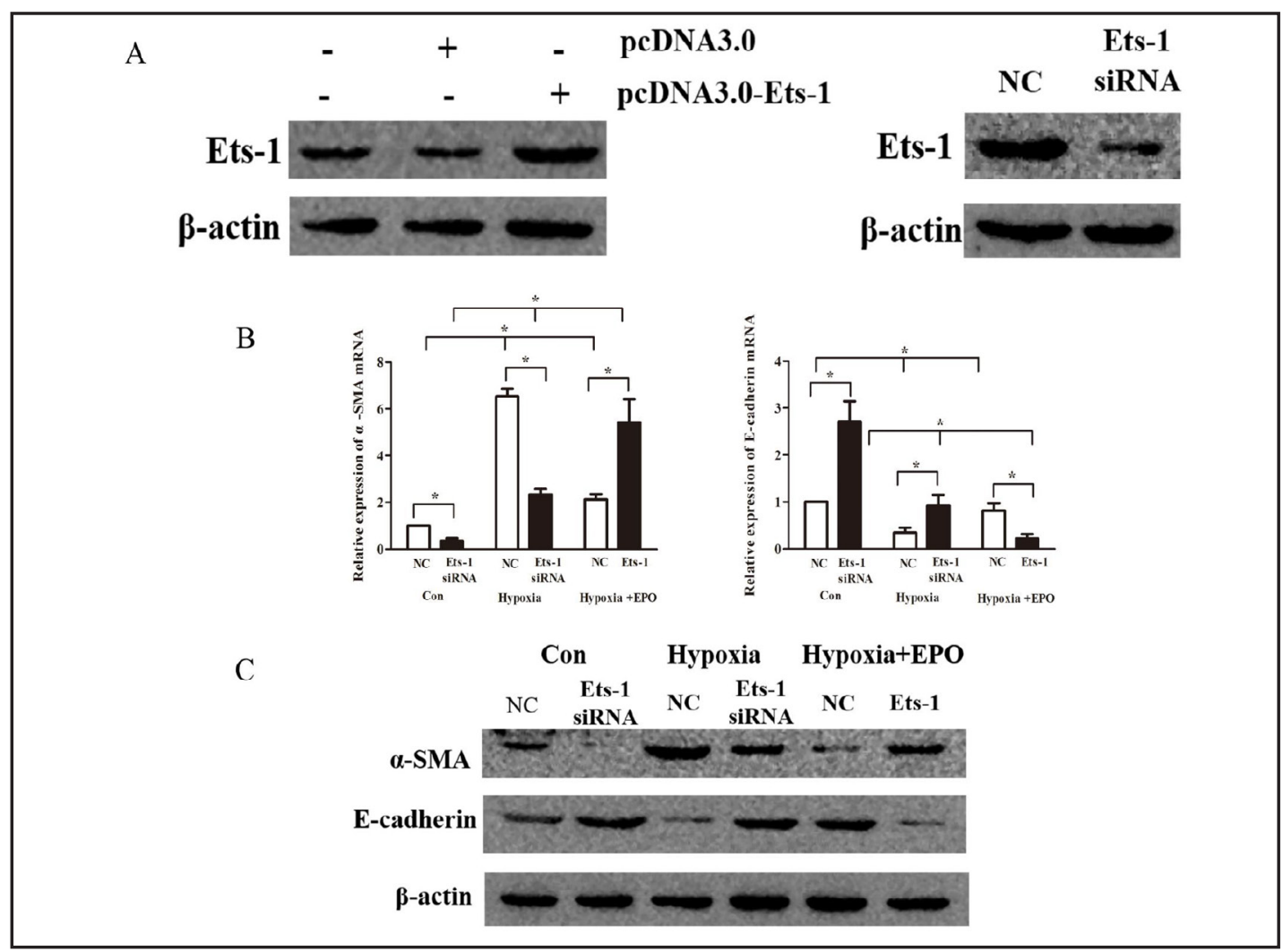

Fig. 4. EPO represses hypoxia-induced EMT by decreasing Ets-1 in HK-2 cells. (A) Western blot analysis of Ets-1 protein expression after transient transfection with expressing plasmid encoding Ets-1 (pcDNA3.0-Ets-1) or transfection with Ets-1 siRNA in HK-2 cells; $\beta$-Actin serves a loading control. (B) Real time PCR analysis of $\alpha$-SMA and E-cadherin in HK-2 cells with hypoxia $\left(1 \% \mathrm{O}_{2}\right)$ treatment with or without EPO $(10 \mathrm{U} / \mathrm{mL})$ for $48 \mathrm{~h}$ in the presence or absence of pcDNA3.0-Ets-1 or Ets-1 siRNA. The data were normalized using a GAPDH control. ${ }^{*} P<0.05$. (C) Western blot analysis of $\alpha$-SMA and E-cadherin in HK-2 cells with hypoxia $\left(1 \% \mathrm{O}_{2}\right)$ treatment with or without EPO $(10 \mathrm{U} / \mathrm{mL})$ for $48 \mathrm{~h}$ in the presence or absence of pcDNA3.0-Ets-1 or Ets- 1 siRNA. $\beta$-Actin serves a loading control.

and the potential role of Ets- 1 after EPO treatment were investigated. Transient expression of Ets-1 in HK-2 cells resulted in a remarkable upregulation of Ets-1 expression (Fig. 4A). Using specific siRNA targeting Ets-1, endogenous Ets-1 expression could be significantly downregulated (Fig. 4A). The expression of E-cadherin increased in cells transfected using Ets-1 siRNA under hypoxic conditions, and was markedly decreased by overexpression of Ets-1 in EPO-treated hypoxic HK-2 cells. The expression of $\alpha$-SMA was reduced in HK-2 cells transfected with Ets-1 siRNA under the same conditions. Transient expression of Ets-1 increased expression of $\alpha$-SMA in EPO-treated cells under hypoxic conditions (Fig. 4B, C).

miR-200b represses the effect of Ets-1-induced EMT by targeting Ets-1

Ectopic expression of Ets-1 promotes EMT. Whether Ets-1 was sufficient to induce EMT in the absence of hypoxia and the role of miR-200 during this process were investigated. Overexpression of Ets- 1 resulted in a significant gain of $\alpha$-SMA and loss of expression of E-cadherin (Fig. 5A-C). Co-transfection of miR-200b with pcDNA3.0-Ets-1 partially restored E-cadherin expression and attenuated $\alpha$-SMA induction (Fig. 5A-C). These results indicate that upregulation of miR-200b is accompanied by downregulation of Ets-1. miR-200b represses the effect of the Ets-1-induced EMT process in HK-2 cells.

Ets- 1 has been proposed as a putative target of miR-200b on the basis of in silico miRNA target prediction programs such as TargetScan and miRanda [17]. In the present study, it was 


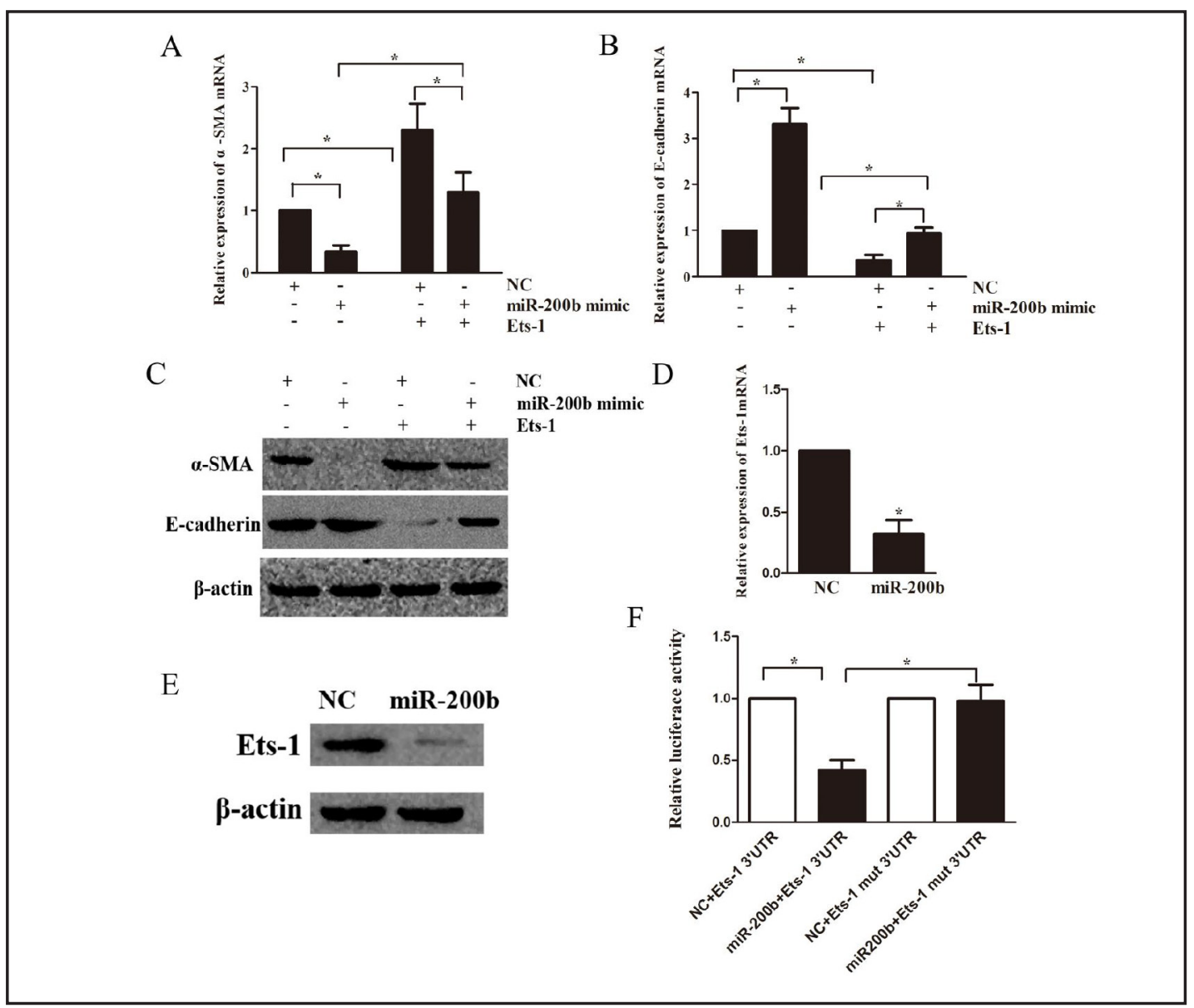

Fig. 5. miR-200b represses the effect of Ets-1-induced EMT by targeting MiR-200b. (A, B) Real time PCR analysis of $\alpha$-SMA and E-cadherin in HK-2 cells which were transfected with miR-200b or NC in the presence or absence of pcDNA3.0-Ets-1. The data were normalized using a GAPDH control. ${ }^{*} P<0.05$. (C) Western blot analysis of $\alpha$-SMA and E-cadherin in HK-2 cells which were transfected with miR-200b or NC in the presence or absence of pcDNA3.0-Ets-1; $\beta$-Actin serves a loading control. (D) Real time PCR analysis of Ets-1 expression in miR-200b mimic-treated HK-2 cells. The data were normalized using a GAPDH control. $* P<0.05$ versus NC. (E) Western blot analysis of Ets-1 protein expression in miR-200b mimic-treated HK-2 cells. $\beta$-Actin serves a loading control. (F) Luciferase reporter assay after miR-200b mimic delivery in HK-2 cells. Open and solid bars represent $\mathrm{NC}$ and miR-200b mimic-treated cells, respectively. Results were normalized using data obtained from an assay with Renilla luciferase and are expressed as mean \pm SEM. $* P<0.05$.

found that Ets-1 expression was markedly reduced by miR-200b in HK-2 cells (Fig. 5D, E). To clarify the interaction between miR-200b and the functional miR-200b seed regions within the 3'UTR region of Ets-1, luciferase reporter assays were performed. Transfection with miR$200 \mathrm{~b}$ resulted in a significant reduction in luciferase activity in HK-2 cells transfected with the wild-type construct of the Ets-1 3'UTR, whereas the luciferase activity of the mutanttype reporter construct remained unchanged by miR-200b mimics (Fig. 5F).

\section{Discussion}

Chronic hypoxia is a crucial process in renal fibrogenesis. The development of renal interstitial fibrosis in CKD is aggravated by chronic tubulointerstitial hypoxia [21]. It is very important to seek new treatment strategies for these conditions. Although specific therapies that inhibit the progression of CKD are unavailable, preventing EMT would be one 


\section{Cellular Physiology Cell Physiol Biochem 2017;42:269-280

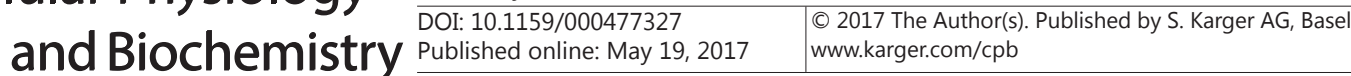 \\ Bai et al.: Erythropoietin Upregulates miR-200b in HK-2 Cells}

Fig. 6. Schematic model depicting EPO control of EMT markers via upregulation of miR-200b mediated repression of Ets-1 expression in HK-2 cells. EPO treatment in HK-2 cells induces the expression of miR-200b. miR-200b counteracts hypoxia-induced EMT via repression of Ets-1.

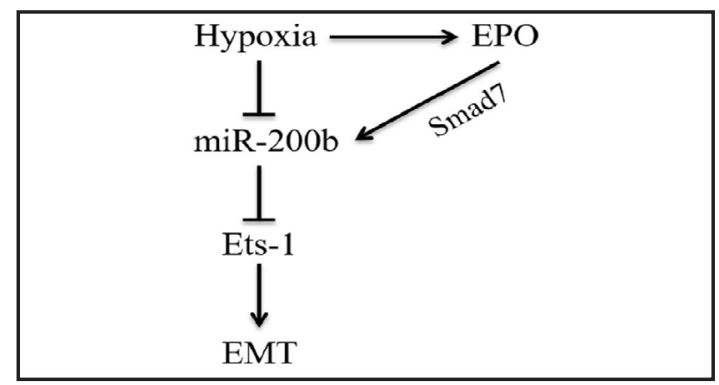

therapeutic option to potentially inhibit renal fibrosis [22]. EPO is a glycoprotein that has a critical hormonal role in regulating erythrocyte production, and it can protect a wide variety of tissues from diverse injuries in addition to its role in erythropoiesis [23]. In this study, it has been shown that EPO attenuates hypoxia-induced EMT in HK-2 cells, and this can be mediated by upregulating miR-200 and suppressing the expression of Ets-1 (Fig. 6). For the first time, we showed that EPO activates the transcription of mir-200 via association with Smad7.

EPO has validated anti-inflammatory, -oxidant, and -apoptotic effects when it is used prior to ischemia. Most studies have focused on EPO preconditioning in kidney ischemic reperfusion injury (IRI). EPO has been shown to reduce glomerular dysfunction and tubular injury, and reduce apoptotic cell death. The anti-apoptotic effects of EPO are dependent on JAK2 signaling [24]. As a widely-used erythropoiesis-stimulating agent in CKD patients, EPO has also recently been used as an antifibrotic agent. EPO treatment protects against chronic renal injury by inhibiting stress induced senescence and EMT in unilateral ureteric obstruction (UUO) mouse model [25]. The current study has demonstrated that EPO represses hypoxia-induced EMT in HK-2 cells, and it subsequently attenuates the expression of $\alpha$-SMA and restores the expression of E-cadherin. EPO treatment produced renal protective effects and inhibited TGF- $\beta$ induced EMT in vivo and in vitro. Previous studies have shown that EPO is likely to counteract TGF- $\beta$ induced EMT by decreasing Smad- 2 phosphorylation [16]. Other studies have indicated that EPO significantly improves renal function, reduces tubular injury and prevents tubular cell apoptosis [26].

miRNAs may play important roles in tubular epithelial cell EMT and renal fibrosis. Previous studies have shown that miR-200 family inhibit EMT through direct targeting of ZEB1 and ZEB2, which encode transcriptional repressors of E-cadherin in kidney tubular cells [27]. miR-200b could ameliorate tubulointerstitial fibrosis by inhibition of EMT of tubular epithelial cells in UUO model mice [28]. The current results demonstrate that miR$200 \mathrm{~b}$ is involved in hypoxia-induced EMT. Hypoxia down-regulates expression of miR-200a, miR-200b and miR-429 in HK-2 cells. Loss of the miR-200 family has been shown to be highly correlated with the mesenchymal phenotype. Recent findings revealed that miR-200b is down-regulated by hypoxia to induce an angiogenic response in endothelial cells [17]. Reexpression of miR-200b blocked hypoxia-induced EMT in lung cancer cells, and knocking down Notch-1 by siRNA under hypoxia resulted in up-regulated miR-200b expressions [29]. Other findings demonstrate that suppression of DICER is sufficient to reduce miR-200 levels and enables cells to undergo EMT in hypoxic human breast cancer cells [30]. miR-200 has been reported to be involved in hypoxic/ischemic events [31]. The current study has, for the first time, indicated that miR-200 mediates the protective effects of EPO in the EMT of HK-2 cells. EPO enhances the beneficial influence of mesenchymal stem cells so that they exert a better restorative effect following TGF- $\beta$ induced fibrosis in HK- 2 cells by upregulating the expression of miRNAs in microvesicles, including miRNA-200 [15]. However, the mechanism underlying EPO regulation of miRNAs remains unclear. The current study has demonstrated that EPO activated miR-200b-a-429 promoter dependent on upregulating Smad7, which can enhance miR-200b-a- 429 promoter activity. Smad7 inhibits the fibrotic effect of TGF- $\beta$ on renal tubular cells by blocking Smad2 activation which provide a therapeutic strategy for renal fibrosis [32]. However, the relationship of Smad7 and miR-200b-a-429 promoter need to be further elucidated. 
Ets-1 is a member of the Ets family of transcription factors that share a unique Ets DNA binding domain. The current study has demonstrated that EPO-induced miR-200 expression attenuates EMT by repressing Ets-1 expression. There are some controversies about the role of Ets-1 in fibroblasts. Ets-1 has been reported to act as a negative regulator of COL1 production and EMT in TGF- $\beta$-stimulated renal cells [33]. Ets- 1 efficiently antagonizes the profibrotic effects of TGF- $\beta$ in human fibroblasts [34]. However, other studies reported that ETS- 1 is a common mediator of the proinflammatory and profibrotic effects of angiotensin II (Ang II)-induced hypertensive renal damage [35]. Knockdown of Ets-1 attenuates Ang II-induced fibroblast activation [36]. Our study favors the view that Ets-1 expression has the profibrotic effect under hypoxia. In the present study, with cultures exposed to hypoxic conditions, it was found that Ets-1 was upregulated after EPO treatment. EPO can protect retinal cells by downregulating hypoxia-inducible factor-1 (HIF-1) expression in diabetic rats [37]. Oikawa reported that hypoxia induces Ets-1 via the activity of HIF-1 [38]. In the present study, it was found that miR-200b is crucial in protecting HK-2 cells from EMT via Ets-1 direct targeting under hypoxic conditions. Previous findings have revealed that hypoxia-sensitive miR-200b is involved in the induction of angiogenesis via directly targeting Ets-1 in human microvascular endothelial cells [17]. Overexpression of miR-200c results in the negative regulation of its gene targets including ZEB1, ETS1 and FLT1, which facilitate gain of epithelial characteristics that are required for mesenchymal-to-epithelial transition behavior in human colorectal cancer cells [39]. This finding suggests that miR200 expression is increased after EPO treatment, which targets and represses Ets-1 in HK-2 cells under hypoxic conditions. EPO may attenuate EMT by regulating HIF-1 and miR-200 in human proximal tubular cells under hypoxic conditions. Further investigation is required to elucidate the underlying mechanism of the EPO induction of miR-200 upregulation.

In conclusion, these data provide evidence that miR-200 mediates the protective effects of EPO on EMT in hypoxic HK-2 cells. EPO attenuated hypoxia-induced EMT in HK-2 cells by increasing miR-200 expression. It is likely to counteract hypoxia induced EMT via repression of Ets-1. These results will help to uncover the possible mechanisms of the EPO renoprotective effect, which could be mediated by regulation of miR-200 in hypoxia-induced EMT.

\section{Acknowledgments}

This work was supported by a grant from the Project of the Army Medical Technology for Youth Training (No. 13QNP002) and National Natural Sciences Foundation of China (No. 81400632). This work was also supported by the Scientific and Technological Foundation of Liaoning Province (No. 2012408002).

\section{Disclosure Statement}

The authors declare no conflicts of interest.

\section{References}

1 Imamura R, Isaka Y, Sandoval RM, Ichimaru N, Abe T, Okumi M, Yazawa K, Kitamura H, Kaimori J, Nonomura N: A nonerythropoietic derivative of erythropoietin inhibits tubulointerstitial fibrosis in remnant kidney. Clin Exp Nephrol 2012;16:852-862.

2 Sun D, Feng J, Dai C, Sun L, Jin T, Ma J, Wang L: Role of peritubular capillary loss and hypoxia in progressive tubulointerstitial fibrosis in a rat model of aristolochic acid nephropathy. Am J Nephrol 2006;26:363-371.

- 3 Sun S, Ning X, Zhang Y, Lu Y, Nie Y, Han S, Liu L, Du R, Xia L, He L: Hypoxia-inducible factor-1 $\alpha$ induces Twist expression in tubular epithelial cells subjected to hypoxia, leading to epithelial-to-mesenchymal transition. Kidney Int 2009;75:1278-1287. 


\section{Cellular Physiology Cell Physiol Biochem 2017;42:269-280

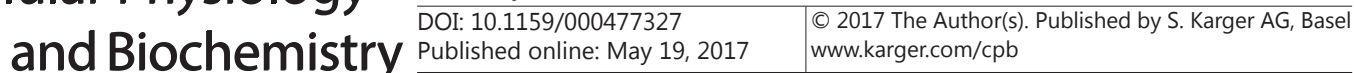

Bai et al.: Erythropoietin Upregulates miR-200b in HK-2 Cells

4 Manotham K, Tanaka T, Matsumoto M, Ohse T, Inagi R, Miyata T, Kurokawa K, Fujita T, Ingelfinger JR, Nangaku M: Transdifferentiation of cultured tubular cells induced by hypoxia. Kidney Int 2004;65:871-880.

5 Higgins DF, Kimura K, Bernhardt WM, Shrimanker N, Akai Y, Hohenstein B, Saito Y, Johnson RS, Kretzler M, Cohen CD: Hypoxia promotes fibrogenesis in vivo via HIF-1 stimulation of epithelial-to-mesenchymal transition. J Clin Invest 2007;117:3810-3820.

6 Hu L, Yang C, Zhao T, Xu M, Tang Q Yang B, Rong R, Zhu T: Erythropoietin Ameliorates Renal Ischemia and Reperfusion Injury via Inhibiting Tubulointerstitial Inflammation. J Surg Res 2012;176:260-266.

7 Ma R, Hu J, Huang C, Wang M, Xiang J, Li G: JAK2/STAT5/Bcl-xL signalling is essential for erythropoietinmediated protection against apoptosis induced in PC12 cells by the amyloid $\beta$-peptide A $\beta 25-35$. Br J Pharmacol 2014;171:3234-3245.

-8 Gong H, Wang W, Kwon TH, Jonassen T, Li C, Ring T, Frøkiær J, Nielsen S: EPO and -MSH prevent ischemia/ reperfusion-induced down. Kidney Int 2004;66:683-695.

-9 Kuriyama S, Tomonari H, Yoshida H, Hashimoto T, Kawaguchi Y, Sakai O: Reversal of anemia by erythropoietin therapy retards the progression of chronic renal failure, especially in nondiabetic patients. Nephron 1997;77:176-185.

10 Srisawat N, Manotham K, Eiam-Ong S, Katavetin P, Praditpornsilpa K, Eiam-Ong S: Erythropoietin and its non-erythropoietic derivative: Do they ameliorate renal tubulointerstitial injury in ureteral obstruction? Int J Urol 2008;15:1011-1017.

11 Lee Y, Ahn C, Han J, Choi H, Kim J, Yim J, Lee J, Provost P, Rådmark O, Kim S: The nuclear RNase III Drosha initiates microRNA processing. Nature 2003;425:415-419.

12 Wang B, Komers R, Carew R, Winbanks CE, Xu B, Herman-Edelstein M, Koh P, Thomas M, Jandeleit-Dahm K, Gregorevic P: Suppression of microRNA-29 expression by TGF- $\beta 1$ promotes collagen expression and renal fibrosis. J Am Soc Nephrol 2012;23:252-265.

-13 Wang B, Koh P, Winbanks C, Coughlan MT, McClelland A, Watson A, Jandeleit-Dahm K, Burns WC, Thomas MC, Cooper ME: miR-200a Prevents renal fibrogenesis through repression of TGF- $\beta 2$ expression. Diabetes 2011;60:280-287.

14 Park S, Gaur AB, Lengyel E, Peter ME: The miR-200 family determines the epithelial phenotype of cancer cells by targeting the E-cadherin repressors. Genes Dev 2009;23:1378.

15 Yan W, Lu X, He J, Zhao W: Influence of erythropoietin on microvesicles derived from mesenchymal stem cells protecting renal function of chronic kidney disease. Stem Cell Res Ther 2015;6:1-14.

16 Park SH, Choi MJ, Song IK, Choi SY, Nam JO, Kim CD, Lee BH, Park RW, Park KM, Kim YJ: Erythropoietin decreases renal fibrosis in mice with ureteral obstruction: role of inhibiting TGF-beta-induced epithelialto-mesenchymal transition. J Am Soc Nephrol 2007;18:1497-1507.

17 Chan YC, Khanna S, Roy S, Sen CK: miR-200b targets Ets-1 and is down-regulated by hypoxia to induce angiogenic response of endothelial cells. J Biol Chem 2011;286:2047-2056.

18 Bai JX, Yan B, Zhao ZN, Xiao X, Qin WW, Zhang R, Jia LT, Meng YL, Jin BQ, Fan DM: Tamoxifen represses miR200 microRNAs and promotes epithelial-to-mesenchymal transition by up-regulating c-Myc in endometrial carcinoma cell lines. Endocrinology 2013;154:635-645.

19 Xiong M, Jiang L, Zhou Y, Qiu W, Fang L, Tan R, Wen P, Yang J: The miR-200 family regulates TGF- $\beta 1$-induced renal tubular epithelial to mesenchymal transition through Smad pathway by targeting ZEB1 and ZEB2 expression. Ajp Renal Physiology 2012;302:F369-379.

-20 Nakerakanti SS, Kapanadze B, Yamasaki M, Markiewicz M, Trojanowska M: Fli1 and Ets1 have distinct roles in connective tissue growth factor/CCN2 gene regulation and induction of the profibrotic gene program. J Biol Chem 2006;281:25259-25269.

21 Mimura I, Nangaku M: The suffocating kidney: tubulointerstitial hypoxia in end-stage renal disease. Nature Reviews Nephrology 2010;6:667-678.

22 Chen CH, Cheng CY, Chen YC, Sue YM, Liu CT, Cheng TH, Hsu YH, Chen TH: MicroRNA-328 inhibits renal tubular cell epithelial-to-mesenchymal transition by targeting the CD44 in pressure-induced renal fibrosis. PLoS One 2014;9:e99802-e99802.

23 Brines M, Cerami A: Discovering erythropoietin's extra-hematopoietic functions: biology and clinical promise. Kidney Int 2006;70:246-250.

24 Sharples EJ, Patel N, Brown P, Stewart K, Mota-Philipe H, Sheaff M, Kieswich J, Allen D, Harwood S, Raftery M: Sharples, E. J. et al. Erythropoietin protects the kidney against the injury and dysfunction caused by ischemia-reperfusion. J Am Soc Nephrol 2004;15:2115-2124. 


\section{Cellular Physiology Cell Physiol Biochem 2017;42:269-280 \begin{tabular}{l|l} 
DOI: 10.1159/000477327 & Ond Biochemistry 2017 The Author(s). Published by S. Karger AG, Basel \\
wuww.karger.com/cpb
\end{tabular}}

Bai et al.: Erythropoietin Upregulates miR-200b in HK-2 Cells

25 Adis Tasanarong SK, Sookkasem Khositseth: Dual Inhibiting Senescence and Epithelial-to-Mesenchymal Transition by Erythropoietin Preserve Tubular Epithelial Cell Regeneration and Ameliorate Renal Fibrosis in Unilateral Ureteral Obstruction. BioMed Research International 2013;2013:308130.

26 Dang J, Jia R, Tu Y, Xiao S, Ding G: Erythropoietin prevents reactive oxygen species generation and renal tubular cell apoptosis at high glucose level. Biomed Pharmacother 2010;64:681-685.

27 Gregory PA, Bert AG, Paterson EL, Barry SC, Tsykin A, Farshid G, Vadas MA, Khewgoodall Y, Goodall GJ: The miR-200 family and miR-205 regulate epithelial to mesenchymal transition by targeting ZEB1 and SIP1. Nat Cell Biol 2008;10:593-601.

-28 Oba S, Kumano S, Suzuki E, Nishimatsu H, Takahashi M, Takamori H, Kasuya M, Ogawa Y, Sato K, Kimura K: miR-200b Precursor Can Ameliorate Renal Tubulointerstitial Fibrosis. PLoS One 2010;5:e13614.

29 Gao XJ, Liu JW, Zhang QG, Zhang JJ, Xu HT, Liu HJ: Nobiletin inhibited hypoxia-induced epithelialmesenchymal transition of lung cancer cells by inactivating of Notch-1 signaling and switching on miR200b. Die Pharmazie 2015;70:256.

30 Van dBT, Koch E, Chu K, Rupaimoole R, Prickaerts P, Adriaens M, Voncken JW, Harris AL, Buffa FM, Haider S: Hypoxia promotes stem cell phenotypes and poor prognosis through epigenetic regulation of DICER. Nature Communications 2014;5:5203-5203.

31 Zhi F, Xue L, Shao N, Deng D, Kang X, Xu Y, Wang R, Yang Y, Xia Y: $\delta$-Opioid Receptor Activation and MicroRNA Expression in the Rat Heart Under Prolonged Hypoxia. Cell Physiol Biochem 2016;39:11181128.

32 Lan HY, Mu W, Tomita N, Huang XR, Li JH, Zhu HJ, Morishita R, Johnson RJ: Inhibition of renal fibrosis by gene transfer of inducible Smad7 using ultrasound-microbubble system in rat UUO model. J Am Soc Nephrol 2003;14:1535.

33 Okano K, Hibi A, Miyaoka T, Inoue T, Sugimoto H, Tsuchiya K, Akiba T, Nitta K: Inhibitory effects of the transcription factor Ets-1 on the expression of type I collagen in TGF- $\beta 1$-stimulated renal epithelial cells. Mol Cell Biochem 2012;369:247-254.

-34 Czuwara-Ladykowska J, Sementchenko VI, Watson DK, Trojanowska M: Ets1 is an effector of the transforming growth factor beta (TGF-beta ) signaling pathway and an antagonist of the profibrotic effects of TGF-beta. J Biol Chem 2002;277:20399.

-35 Feng W, Chumley P, Hua P, Rezonzew G, Jaimes D, Duckworth MW, Xing D, Jaimes EA: Role of the Transcription Factor Erythroblastosis Virus E26 Oncogen Homolog-1 (ETS-1) as Mediator of the Renal Proinflammatory and Profibrotic Effects of Angiotensin II. Hypertension 2012;60:1226-1233.

36 Di J, Jiang L, Zhou Y, Cao H, Fang L, Wen P, Li X, Dai C, Yang J: Ets-1 Targeted by MicroRNA-221 Regulates Angiotensin II-Induced Renal Fibroblast Activation and Fibrosis. Cell Physiol Biochem 2014;34:1063-1074.

37 Xu G, Kang D, Zhang C, Lou H, Sun C, Yang Q Lu L, Xu GT, Zhang J, Wang F: Erythropoietin Protects Retinal Cells in Diabetic Rats Through Upregulating ZnT8 via Activating ERK Pathway and Inhibiting HIF-1 $\alpha$ Expression. Invest Ophthalmol Vis Sci 2015;56:8166-8178.

38 Oikawa M, Abe M, Kurosawa H, Hida W, Shirato K, Sato Y: Hypoxia Induces Transcription Factor ETS-1 via the Activity of Hypoxia-Inducible Factor-1. Biochem Biophys Res Commun 2001;289:39-43.

-39 Hur K, Toiyama Y, Takahashi M, Balaguer F, Nagasaka T, Koike J, Hemmi H, Koi M, Boland CR, Goel A: MicroRNA-200c modulates epithelial-to-mesenchymal transition (EMT) in human colorectal cancer metastasis. Gut 2013;62:1315-1326. 